\title{
La Feria Lúdico-Pedagógica como espacio de reflexión curricular
}

\author{
Amparo Jaimes \\ Facultad de Educación, Licenciatura en Educación Infantil, Corp. Universitaria Minuto de Dios, Bogotá, Colombia. \\ (correo-e: amparo.jaimes@uniminuto.edu.co; ajaimes@uniminuto.edu.co)
}

Recibido Nov. 6, 2019; Aceptado Ene. 6, 2020; Versión final Mar. 27, 2020, Publicado Ago. 2020

\begin{abstract}
Resumen
En este estudio se expone la Feria Lúdico Pedagógica como herramienta práctica para proponer estrategias de fortalecimiento del programa de Licenciatura en Pedagogía Infantil. Se desarrolló un proceso de interpretación hermenéutica del contenido de los proyectos escritos y del material lúdico resultado de las prácticas desarrolladas en la ejecución de la Feria Lúdica. Se utilizó una muestra de 80 estudiantes de la Licenciatura. El análisis mostró que esta actividad aporta elementos prácticos para mejorar las relaciones y los métodos de enseñanza-aprendizaje en la formación de educadores y en el trabajo con primera infancia. Se concluye que la lúdica es una herramienta de aprendizaje que permite analizar situaciones para entender a cabalidad el rol del docente en el proceso formativo del individuo y la manera como la universidad aporta a la construcción de personalidades de educadores que respondan a las necesidades de la sociedad actualmente.
\end{abstract}

Palabras clave: lúdica; primera infancia; pedagogía; didáctica; reflexión curricular

\section{The Leisure and Pedagogical Fair as a space for curricular reflection}

\begin{abstract}
The Playful Pedagogical Fair is presented as a practical tool from which to propose strategies for strengthening the Bachelor's Degree program in Children's Pedagogy. The present study developed a process of contextbased hermeneutic interpretation of the written projects and the recreational material retrieved from the practices developed in the execution of the Playful Pedagogical Fair. The study's sample consisted of 80 bachelor's degree students. The results showed the Playful Pedagogical Fair provides practical elements to improve relations and teaching-learning methods for the training of educators and for early-childhood work. In conclusion, ludic play is a learning tool that enhances analytical thinking for complete understanding of a given situation. This includes the role of the teacher in the training process of the individual and the way a university contributes to the shaping of educators' personalities that respond to the needs of today's society.
\end{abstract}

Keywords: playful; early childhood; pedagogy; didactic; curricular reflection 


\section{INTRODUCCIÓN}

En el marco de los procesos de formación del Programa de Licenciatura en Pedagogía Infantil, desde el año 2009, se realiza una "Feria lúdico- pedagógica" la cual se configura como una estrategia de socialización de los procesos y aprendizajes alcanzados en el desarrollo de la práctica pedagógica desarrollada por los estudiantes y que hace parte del currículo del programa, generando a su vez ideas y cuestionamientos que suscitan a la reflexión en torno a los procesos pedagógicos generados con su implementación. De acuerdo con lo anterior, para el desarrollo de la investigación que expone el presente artículo, se planteó el estudio y reflexión sobre la práctica pedagógica y su influencia en los procesos formativos, teniendo como objetivo principal: Reconocer la Feria lúdico Pedagógica como una estrategia de fortalecimiento, articulación y pertinencia curricular del programa de Licenciatura en Pedagogía Infantil de la Corporación UNIMINUTO.

Para el desarrollo de la investigación se plantearon también una serie de objetivos específicos que guiaron el proceso, para un análisis crítico de la feria lúdico - pedagógica y su aporte a los procesos formativos del programa; producir nuevos significados pedagógicos teórico- prácticos que generen impacto social a través de la práctica en la feria lúdico pedagógica dirigida a infancias, y, por último, fortalecer las competencias de enseñanza - aprendizaje de los estudiantes. El estudio de los alcances y aportes de la Feria que desarrollan los estudiantes de la licenciatura, resultó propicio para establecer la pertinencia de continuar ejecutando dichos procesos, reconocer estrategias para fortalecer la praxis educativa, y además identificar significados que dinamicen el programa de Licenciatura en Pedagogía Infantil.

En este sentido, la investigación se enmarcó en la importancia de pensar la feria lúdico - pedagógica, como un espacio propicio para reflexionar sobre los métodos, los significados y la didáctica, elementos inmersos en los procesos educativos y de formación en la infancia, pero además de analizar críticamente los aprendizajes de los estudiantes de la licenciatura. Bajo estas condiciones, el análisis en torno a la feria lúdico -pedagógica, permitió ahondar además en las competencias a desarrollar en los individuos en proceso de estudio de futuros profesionales de la Educación, comprendiendo además que la coherente formación de educadores de etapa infantil aporta en gran manera al desarrollo integral de la primera infancia.

Mediante una revisión de literatura sobre el tema en cuestión, se hicieron hallazgos de trabajos investigativos previos, que abordaban aspectos como: la lúdica y la pedagogía en el marco del aprendizaje y el espíritu científico en primera infancia; la importancia de la lúdica en la creatividad y la dimensión afectiva de los niños; además de la lúdica y su relación con el currículo de educación infantil. Un primer trabajo corresponde al realizado por Moya et al., (2015), titulado: "La Educación Infantil que queremos: investigaciones y experiencias", en este trabajo se presentan una serie de propuestas que invitan a reconocer al niño en su contexto: familiar, escolar y social, ya que "el aula Infantil debe ser un espacio vital en el que se aprende, se educa, se participa, se imagina, se ríe, se crea y se sueña", las autoras abordan la importancia de reconocer la Educación Infantil como una etapa escolar en la vida de los niños pero a "además, en ella se inician varios procesos exclusivamente humanos como son el desarrollo intelectual, el de unas habilidades y destrezas físicas y cognitivas, el de un rico mundo interior de afectos y sentimientos; en pocas palabras: comienza el proceso de crecimiento personal en todas sus dimensiones: racional, biológica, sensorial, estética, afectiva, creadora y moral" (p. 2)

Este trabajo aportó elementos teóricos que explican la importancia de reconocer la Educación Infantil como una etapa en la que se desarrollan procesos de aprendizaje en las diversas dimensiones de los niños, además resalta la importancia de integrar la lúdica en los procesos de enseñanza- aprendizaje, demostrando que las actividades de este tipo dotan al maestro de herramientas didácticas creativas que además de facilitar la transmisión de conocimiento, permiten al maestro de primera infancia dinamizar sus clases y promover la participación activa por parte de los niños con actividades que respondan a las necesidades y capacidades de los estudiantes haciendo de la labor formativa un proceso inclusivo y heterogéneo.

Por otro lado, se evidencia la investigación desarrollada por Tresserras (2017) titulado: "Aportaciones a la formación del profesorado a través del análisis de una experiencia de arte y juego dramático en el primer ciclo de educación infantil" de la Universidad del País Vasco, España, en donde la autora desarrolla una experiencia de vivencia y reflexión a la actividad lúdica del teatro para crear un nexo entre las artes escénicas, la escuela infantil, y la formación inicial del profesorado, es decir, entre lo pedagógico y lo artístico, entre la cultura infantil y la adulta, con el fin de "comprender e interpretar la realidad educativa; analizar los pilares pedagógicos en los que se apoya esta actividad lúdica; y valorar si ofrece recursos para innovar y experimentar tanto en la formación del profesorado, como en la educación infantil". En esta propuesta la autora aborda elementos pedagógicos tanto para la educación infantil como para la formación de profesores de preescolar, dentro de los cuales aborda "temáticas de reciente urgencia para la comunidad educativa como son la escolarización temprana, la educación artística en infantil, la alfabetización audiovisual y tecnológica en edades tempranas, y las metodologías activas, cooperativas y vivenciales para una formación del profesorado centrada en la 
adquisición de competencias (sociafectiva, expresiva y comunicativa, experiencial y lúdica, e identitaria y cultural)" (p. 21)

La anterior propuesta demostró la importancia pedagógica de la lúdica para propiciar espacios para la reflexión y a su vez herramientas didácticas para los profesores, y expone la necesidad de aportar a los futuros docentes en estrategias que dinamicen sus procesos de enseñanza motiven y despierten interés en los niños, permitan explorar sus capacidades y estimular la imaginación generando aprendizajes significativos, exigiendo al docente el diseño e implementación adecuados para alcanzar mejores resultados.

Por su parte, Cáceres et al. (2018), de la Universidad Católica del Maule de Chile, realizaron una investigación titulada "Inclusión y Juego en la Infancia Temprana", en la que proponen profundizar teóricamente el concepto de juego a fin de favorecer procesos de inclusión y aprendizaje en primera infancia "en niños con desarrollo típico o aquellos que presentan retrasos en el desarrollo o discapacidad". Los autores proponen el diseño de un programa de acompañamiento y desarrollo del juego, que considera las fortalezas y debilidades de cada niño, reconociendo los aspectos afectivos y socioemocionales como elementos centrales en el desarrollo de habilidades a partir del juego. De esta manera los autores concluyen que: "las actividades lúdicas como estrategia pedagógica en educación inicial fomentan en los niños y niñas un conjunto de valores éticos y morales que se traducen en espontaneidad, socialización e integración". (p. 189). Este estudio demuestra que la implementación pedagógica del juego, permite el mejoramiento de la formación del escolar, su sociabilidad, su creatividad, su aprendizaje y la satisfacción de la necesidad lúdica. Este estudio aportó a la investigación en el sentido que expone y fundamenta la necesaria integración del juego a los currículos de primera infancia, como u medio de inclusión y reconocimiento de la diversidad de los niños, identifica sus habilidades, fortalezas y debilidades, a la vez que expone la lúdica y el juego como herramientas de apoyo de los profesionales para el desarrollo y mejoramiento de sus prácticas de aula.

La investigación desarrollada por Santos et al. (2019), "Aplicación de actividades lúdicas artísticas para el desarrollo del lenguaje de los niños y niñas", es una propuesta que centra la investigación en la didáctica, donde la lúdica hace parte del currículo escolar, así las autoras proponen el mejoramiento de las habilidades de lenguaje en la formación escolar de los niños y niñas, mediante la utilización de actividades lúdico creativas. Ellas exponen que: "El arte y las actividades lúdicas son parte fundamental en el desarrollo del lenguaje y la formación académica del estudiante. Las actividades lúdicas se han evidenciado desde la existencia del ser humano, por medio de los juegos, el infante va descubriendo el significado de las cosas. Jugar es un método y un medio fundamental innato en el ser humano para exponer sus emociones e intereses sociales" (p. 8).

Por último, Samacá (2015), en un trabajo titulado "El espríritu científico en la Primera Infancia", reflexiona sobre el "espíritu científico" de los niños, explica que en pedagogía es necesario pensar en la manera como se desarrolla y funciona su lógica, la necesidad de "reconocer en el medio sociocultural las posibilidades para estimular talentos o las limitantes que demarcan su desarrollo y, por otra, en la práctica pedagógica, para establecer escenarios de diálogo con los escolares con el fin de leer sus necesidades e intereses y orientar sus búsquedas" En su escrito la autora afirma que: "El juego y la lúdica son elementos determinantes en el quehacer pedagógico, pues le exigen al maestro un ejercicio hermenéutico y fenomenológico que deviene de la interacción entre los distintos lenguajes de los cuales se vale el infante para comunicar sus pensamientos, emociones e ideas" (p. 89).

Este antecedente, expone la capacidad de investigación, la habilidad de descubrir y la curiosidad como elementos fundamentales a lo largo de la vida, y los cuaesl se fundamentan en la infancia, y que, de acuerdo con la autora, es necesario desarrollar y reforzar en los procesos de enseñanza - aprendizaje, y además presenta la actividad lúdica y creativa como una herramienta de gran utilidad para gestionar estas destrezas innatas en los individuos, las cuales se pueden propiciar mediante el diseño de juegos que exijan la capacidad indagatoria de los niños.

De esta manera, partiendo de lo argumentado por los trabajos anteriores, existe una necesidad latente de integrar la lúdica con los currículos, con el fin de dinamizar la práctica educativa para aportar a la formación de la persona desde sus primeros años y para formar educadores integrales. Este aspecto es claramente un elemento que exige ser analizado a profundidad para comprender los procesos de enseñanza- aprendizaje y la manera como desde el programa de Pedagogía Infantil, se están dando a los estudiantes las herramientas pedagógicas necesarias, suscitando evidentemente la observación y consecuente debate dentro de la academia para evaluar la verdadera y significativa enseñanza, generando espacios de discusión para los maestros en formación, puesto que la educación de los niños depende de su correcto desarrollo.

En este sentido se abordaron los conceptos de currículo, lúdica, praxis educativa y pedagogía como elementos teóricos fundamentales para el análisis y reflexión en torno a la feria lúdico-pedagógica del Programa de Licenciatura en Pedagogía Infantil. La lúdica es un medio que permite el compartir y aprehender con la otredad, como bien lo plantea Vigotsky (1933) citado por Lopez, (2017), "el juego surge como necesidad 
de reproducir el contacto con lo demás, es una actividad social, en la cual, gracias a la cooperación con otros niños, se logran adquirir papeles o roles que son complementarios al propio". este concepto es importante para la investigación que evalúa la necesidad de reconfigurar la concepción de la lúdica y el juego como estrategias de formación.

El docente de educación inicial debe desarrollar la actividad lúdica como estrategia pedagógica buscando dar respuesta satisfactoria a la formación integral del niño y la niña, para generar categorías de pensamiento y aportar a la construcción de imaginarios y conocimientos que los niños puedan aplicar en su realidad, como bien expone Piaget (1962) citado por López (2017) "el juego forma parte de la inteligencia del niño, porque representa la asimilación funcional o reproductiva de la realidad según cada etapa evolutiva del individuo". Broadhead et al. (2010), afirman que "si bien la palabra "juego" se usa en los documentos y políticas de los primeros años, no está ni claramente definida ni bien entendida por los profesionales o los responsables de la formulación de políticas. La palabra "juego" se usa a la ligera para significar cualquier cosa que se emprenda "juguetonamente", ya sea que los niños desarrollen las actividades elegidas por ellos mismos o la enseñanza lúdica decidida por parte de los adultos" (p. 11). De acuerdo con lo anterior, es necesario que en los contextos escolares, el juego sea aceptado como un enfoque legítimo para el aprendizaje de los niños a lo largo de sus primeros años a través de la pedagogía de la educación de la primera infancia, comprendiéndolo como una herramienta pedagógica que exige planeación y estar enfocado hacia el aprendizaje, el docente debe guiar los procesos pero es fundamental la libertad en el momento de su desarrollo con la conciencia del juego como un camino y el conocimiento como una consecuencia de su ejecución adecuada.

Las capacidades sensorio motrices, simbólicas o de razonamiento, como aspectos esenciales del desarrollo del individuo, son las que condicionan el origen y la evolución del juego. De acuerdo con Ausubel (1983) citado por Sánchez (2016) "El juego es una actividad necesaria del ser humano, siendo una herramienta útil para adquirir y compartir habilidades intelectuales motoras o afectivas. Que se convierte en una herramienta de aprendizaje significativo en el aula" Claramente esta concepción de lúdica se articula a la comprensión de la praxis pedagógica, la lúdica se resignifica como un elemento útil en la vida y que genera espacios de disfrute y goce, en las que el docente diseña y ejecuta actividades que construyen imaginarios y estimulan competencias mediante el juego planeado, características fundamentales de las ferias lúdicas.

Cuando se abordó la necesidad de fortalecer las competencias de enseñanza-aprendizaje del programa en Pedagogía Infantil a través del desarrollo de la Feria Lúdico -pedagógica como espacio de deconstrucción, se hacía referencia a la transformación paradigmática del aprendizaje tradicional, dándole protagonismo a la innovación en el aula que en apartados anteriores se expone, para lo que es importante comprender la importancia de las competencias, que Gardner (2017) presenta como "un saber- hacer especifico determinado por la cultura y la sociedad", tanto de los docentes en proceso de formación como de los niños y niñas a quienes dirigen sus prácticas". Y es que en el aula de preescolar sin duda alguna el maestro se encuentra con el gran reto de derrumbar estructuras inoperantes, de estimular las habilidades de sus estudiantes y ejercitar las propias: la capacidad de resolver problemas y preguntarse por el funcionamiento de la realidad, elementos de análisis presentes a lo largo de la vida, que revisten gran complejidad y los cuales es necesario afianzar en un sentido lúdico- pedagógico organizado, que como explica Chomsky (1965) citado por Sánchez (2016), debe ir acompañado de "un conjunto de reglas más o menos refinadas que permiten la generación de innumerables desempeños", competencias analizadas en los estudiantes del Seminario de Infancia. En este sentido es importante la creación de relaciones con los niños a través del juego. Una estrategia didáctica centrada en el niño, que destaca la importancia de comprender el mundo y la perspectiva del niño para planear e implementar la actividad lúdica en el aula. Este enfoque facilita los procesos de aprendizaje mientras permite que el docente y los niños se conecten completamente (Landreth, 2012)

Por otra parte, en todo este proceso investigativo, subyace el currículo, como un elemento de vital importancia pues a partir de este se diseñan y organizan todos los elementos anteriores. Según Sacristán (1998), citado por Alvarado (2019) "concibe al currículo como un proceso dinámico de adaptación al cambio social en general y al sistema educativo en particular" pues este responde a las necesidades educativas, sociales, económicas y culturales de las comunidades educativas. El currículo es el espacio complejo y organizado que contiene las temáticas, los símbolos, las prácticas, los métodos y estrategias que dirigen los procesos educativos y que fundamentan los objetivos fundamentales de la educación en todos los niveles, por lo que el hecho de reflexionar en torno a la Feria lúdico - pedagógica es además analizar críticamente sobre el currículo mismo del programa de Pedagogía Infantil para comprender su significación educativa, social, económica y cultural.

Con relación a la concepción de la práctica pedagógica Huberman (1998) citado por Tapia y Muñoz (2019), expone la práctica profesional como: "Proceso consciente, deliberado, participativo implementado por un sistema educativo o una organización con el objeto de mejorar desempeños y resultados, estimular el desarrollo para la renovación en campos académicos, profesionales o laborables y formar el espíritu de compromiso de cada persona con la sociedad y particularmente para con la comunidad en la cual se desenvuelve". 
Es importante, además, reflexionar sobre las políticas que abordan el juego y la relación necesaria en todos los contextos en la familia con los padres y en los medios sociales, reconociendo las influencias culturales y la comprensión del juego como estrategia de enseñanza- aprendizaje. Es una invitación a considerar cómo se puede integrar el juego en el currículo en diversos contextos, para explorar y reflexionar críticamente sobre la noción de juego, considerando la construcción del juego en las políticas educativas. En torno a esto, Wood (2013) evalúa "el papel del juego en el aprendizaje y desarrollo de los niños y desafía el discurso de la libre elección y el juego libre en la educación de la primera infancia, estableciendo el juego como un espacio de atención de los niños, autorregulación y control, así como una oportunidad para explorar sus mundos" (p. 8)

De esta manera, la investigación en torno a La Feria Lúdico pedagógica, comprendió un ejercicio evaluativo a nivel curricular, pedagógico y disciplinar del Programa de Licenciatura en Pedagogía Infantil, que profundizó en aspectos fundamentales de la Educación y es una invitación a la reflexión sobre la manera como se están formando los educadores de primera infancia. Resalta la importancia de abordar desde la academia el reconocimiento de las diversas formas de aprendizaje de los niños y las niñas, con el propósito de facilitar procesos de enseñanza y renovar las prácticas y dinámicas educativas. Siendo la reflexión curricular y de las acciones pedagógicas un proceso continuo y el sustento de los programas de formación docente.

\section{METODOLOGÍA}

La investigación en torno a la Feria Lúdico Pedagógica, pretendió la interpretación de los significados que se evidencian en su desarrollo, los cuales contribuyeron al análisis del Programa en Pedagogía Infantil, con miras a brindar aportes teóricos que enriquecieran y fortalecieran los procesos de enseñanza- aprendizaje de la comunidad educativa en las diferentes regiones. El estudio se desarrolló desde una metodología cualitativa, de tipo exploratorio y con un enfoque hermenéutico el cual aportó elementos fundamentales con los cuales interpretar y comprender los motivos internos de la acción humana, en medio de procesos libres y sus expresiones presentadas textualmente. Es el método hermenéutico precisamente un proceso de interpretación de textos que pretende comprender la experiencia humana evidenciada de forma escrita. Se abordan además aspectos epistemológicos y pedagógicos del enfoque fenomenológico-hermenéutico de investigación educativa de Van Manen (2003) citado por Galvis (2018) "Este enfoque se orienta a la descripción e interpretación de las estructuras esenciales de la experiencia vivida, así como al reconocimiento del significado e importancia pedagógica de esta experiencia".

Así, se desarrolló un proceso de decodificación e interpretación (descriptiva y reflexiva) del contenido de los proyectos escritos y el material lúdico, evidencias de las practicas desarrolladas por los estudiantes de segundo semestre, los cuales hacen parte de la ejecución de la Feria Lúdico Pedagógica como experiencia a partir de la cual se pretendió traducir, interpretar y comprender los mensajes y significados de los productos que fueron resultado de las 10 ferias y desde donde se pretende analizarla como un espacio de reflexión curricular. Mediante el estudio fenomenológico- hermenéutico, se analizó "el significado esencial de los fenómenos, así como el sentido y la importancia que éstos tienen [...] el interés se orienta a la determinación del sentido y la importancia pedagógica de los fenómenos educativos vividos cotidianamente [...] para comprender la naturaleza del conocimiento —-pedagógico, en este caso- y su vinculación con la práctica" (Van Manen, 2003 citado por Galvis, 2018). Esta investigación se desarrolló en la Corporación Universitaria Minuto de Dios, Institución de Educación Superior, de modalidad presencial, virtual y a distancia tradicional, ubicada en sector urbano. Los participantes fueron 80 estudiantes de la Licenciatura en Pedagogía Infantil, seleccionados de manera aleatoria.

En el desarrollo de la investigación, se determinaron las categorías a interpretar como unidades de información, a partir de las cuales abarcar el análisis y reflexión de los textos y materiales lúdicos. Se tomaron como unidades de análisis: lúdica, currículo y políticas públicas de Primera infancia, seleccionadas por su importancia dentro del contexto educativo y las prácticas pedagógicas. Además, se pretendió estudiar la formación del Licenciado en Pedagogía infantil, por lo que se delimitaron además una serie de sub categorías como fueron: pertinencia pedagógica, experiencia (práctica pedagógica), articulación, impacto social, competencias y deconstrucción, las cuales constituyen vital importancia dentro de las prácticas de los estudiantes en la ejecución de la Feria Lúdico Pedagógica. En este sentido el análisis hermenéutico se organizó (Tabla 1), partiendo de los propuesto por Van Manen (2003) citado por Galvis (2018) en tres etapas:

La información se codificó y organizó en forma de tablas, en las que se detallaron los hallazgos encontrados en los trabajos escritos y el material lúdico, y el consecuente análisis y reflexión en torno a las unidades y categorías seleccionadas, para reflexionar en torno a la pertinencia curricular del Programa de Pedagogía Infantil y dar aportes para el fortalecimiento de los contenido y prácticas de la licenciatura para lanzar proyecciones sobre la Educación. En este sentido, el análisis hermenéutico textual de los trabajos escritos, el material didáctico y las acciones de los estudiantes en el desarrollo de sus prácticas pedagógicas, tuvo por finalidad describir situaciones, concepciones y percepciones dadas en las Ferias Lúdico Pedagógicas desarrolladas. 
Tabla 1: Proceso metodológico, La Feria Lúdico Pedagógica como espacio de reflexión curricular (Adaptada de Galvis 2018)

\begin{tabular}{|l|l|l|}
\hline Etapa & \multicolumn{1}{|c|}{ Contenido } & \multicolumn{1}{|c|}{ Actividades } \\
\hline I :Descripción & $\begin{array}{l}\text { Recolección y organización de las } \\
\text { evidencias y experiencias. }\end{array}$ & $\begin{array}{l}\text { Trabajos escritos/ proyectos de aula. } \\
\text { Evidencia fotográfica. Organización de } \\
\text { las unidades y subcategorías de análisis. } \\
\text { Detección de conceptos presentados en } \\
\text { los trabajos escritos y demás evidencias, } \\
\text { para interpretar las relaciones entre sí. } \\
\text { Análisis y reflexión de los hallazgos } \\
\text { encontrados. Reescritura crítica. }\end{array}$ \\
\hline
\end{tabular}

\section{RESULTADOS}

A continuación, se presentan los resultados del análisis de los datos obtenidos de la investigación. Estos resultados demuestran los hallazgos encontrados en los trabajos escritos, el material didáctico y demás evidencias (fotografías, videos) de las Ferias Lúdico Pedagógicas objeto de investigación. Destacan las unidades y subcategorías de análisis como ejes descriptivos y de reflexión textual las cuales facilitaron la organización y la interpretación de los significados y evidencias pedagógicas subyacentes en las prácticas pedagógicas de los estudiantes en el marco de la Feria Lúdico Pedagógica.

Mediante la exploración desarrollada, se establecieron elementos epistemológicos en los temas pedagógicos analizados, posibilitando abordar a profundidad en aspectos concernientes al paradigma humanista y fenomenológico implícitos en los documentos redactados por los estudiantes y sus acciones como actores educativos en el desarrollo de la Feria Lúdico Pedagógica.

Como se indicó con anterioridad, para el análisis hermenéutico se siguieron las etapas de descripción, interpretación y reflexión (Galvis, 2018) las cuales permitieron establecer relaciones conceptuales, se determinaron códigos comunes entre los trabajos escritos de los estudiantes, se ordenaron los datos, se compararon los hallazgos de las evidencias, para llegar a la reflexión sobre los elementos subjetivos inmersos en las estructuras discursivas de los estudiantes, para determinar la manera como los conceptos aprendidos a lo largo de la formación, se evidenciaban tanto en la teoría como en la práctica pedagógica en el marco de la Feria Lúdico Pedagógica, objeto de investigación.

\section{Etapa de descripción}

En el proceso de descripción, se partió de la recolección y organización de las evidencias y experiencias, mediante las cuales se definieron las unidades o categorías de análisis principales: Lúdica, Currículo, Políticas Públicas, y se relacionaron los aspectos destacados en los trabajos escritos y evidencias fotográficas. Se determinaron los conceptos con los que los estudiantes relacionan las unidades de análisis (Fig. 1, 2 y 3). Con esta información, se establecieron diagramas que representan los conceptos con los que los estudiantes las relacionan. En la mayoría de los trabajos escritos y las evidencias fotográficas, los estudiantes relacionan la lúdica con el juego, la asumen como una estrategia para la enseñanza y promueve la imaginación. Los trabajos escritos evidencian que los estudiantes definen el currículo como un elemento de doble significancia. Por un lado, lo asumen como un elemento dinámico que integra la subjetividad y los intereses de los sujetos partícipes en el acto educativo. Por otra parte, definen el Currículo como un elemento estático, de exigencia y que compendia los contenidos de las diversas áreas del conocimiento.

En esta etapa se dio un segundo momento, en el que se determinaron subcategorías con las cuales se facilitó la abstracción y clasificación de la información de los trabajos escritos y evidencias fotográficas de los estudiantes, y a fin de profundizar en su interpretación y responder a cuestionamientos como: ¿Qué relaciones de articulación se dan en la práctica pedagógica?, ¿Cuál es el impacto social de la Lúdica?, ¿Cómo es la experiencia de la Feria lúdico Pedagógica?, ¿Cuál es el valor pedagógico de la Feria lúdica?, entre otros. A partir de la información obtenida se establecieron las definiciones más relevantes y manteniendo como elemento fundamental las vivencias, la experiencia en torno a la práctica pedagógica de la Feria Lúdica.

Los estudiantes relacionan esta unidad de análisis como un elemento que implica otros conceptos importantes en el ámbito de la Educación y con el cual se encuentra profundamente relacionado y lo asumen como un derecho de la infancia y posibilita la inclusión. 


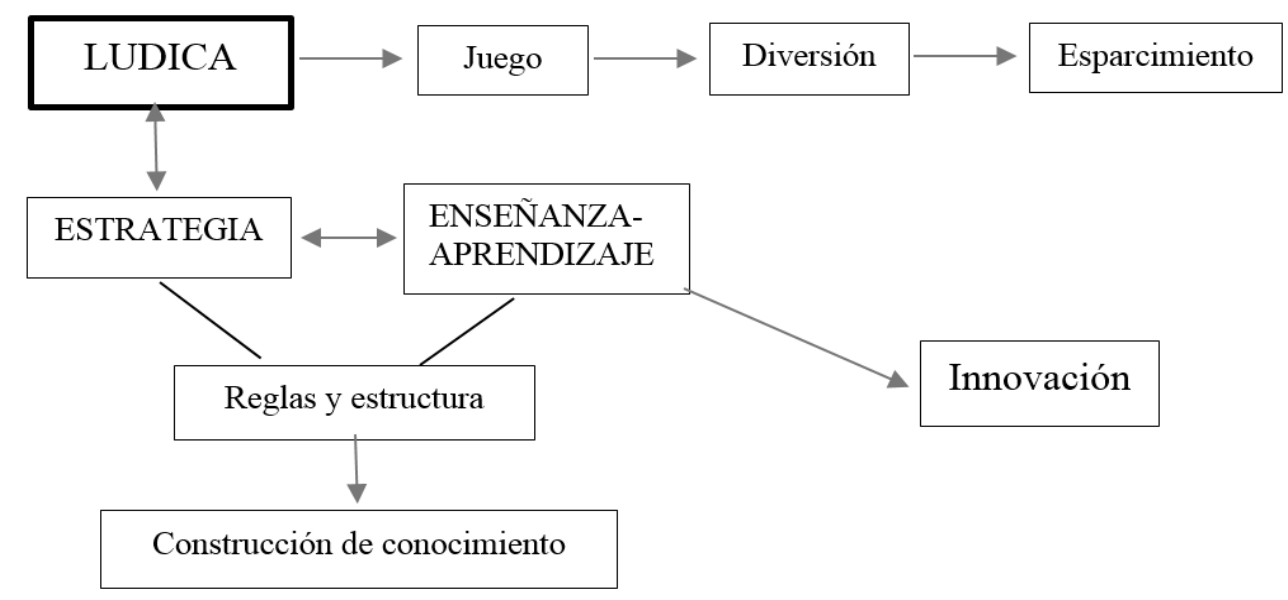

Fig. 1: Relación de conceptos en el marco de la Lúdica.

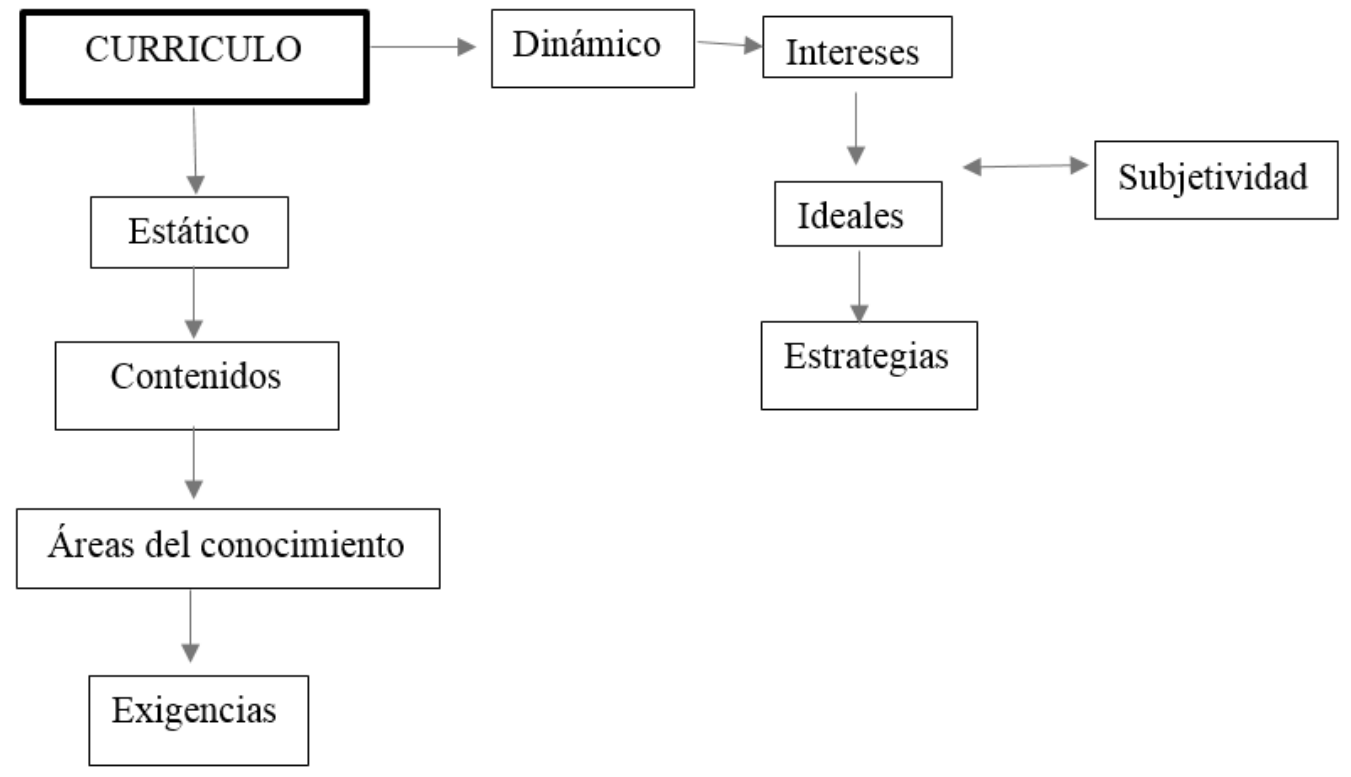

Fig. 2: Representación concepto Currículo.

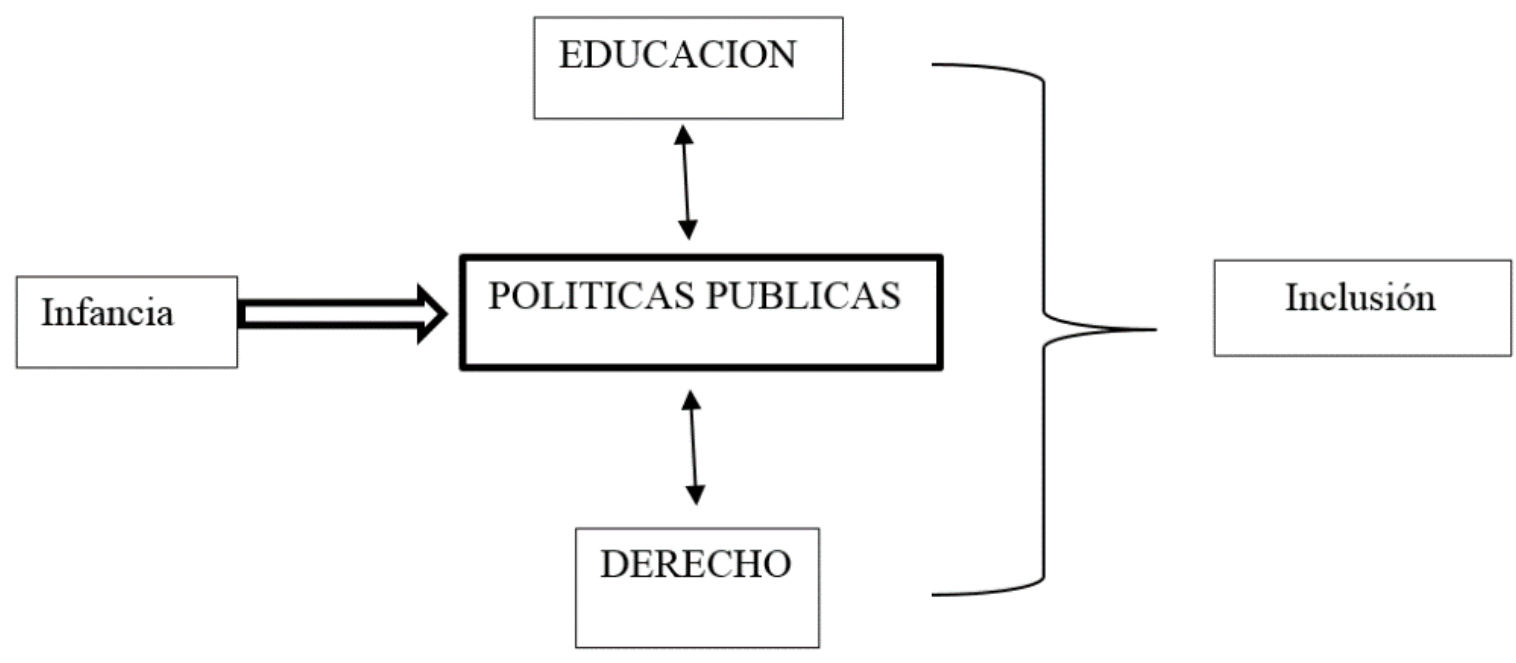

Fig. 3: Representación concepto Políticas Públicas. 


\section{Etapa de Interpretación}

En esta etapa el trabajo se centró en la interpretación del material experiencial (trabajos escritos y evidencia fotográfica) con el objetivo de determinar conceptos y relaciones temáticas en torno a las unidades y subcategorías de análisis. Para esto se realizó un análisis temático, con el que se determinaron los significados fundamentales dentro de las ideas expuestas en los trabajos de los estudiantes para establecer su relación con las unidades y subcategorías de análisis y su aporte al análisis crítico objetivo de la investigación. Este análisis orientado al significado implícito en las prácticas pedagógicas y cognitivas planteadas por los estudiantes. El siguiente fragmento representa el proceso anteriormente descrito:

Los niños que ingresan a un entorno educativo, muestran reacciones adversas al medio que lo componen (maestros, estudiantes, nuevos lugares, etc.), debido al cambio abrupto que adquieren. Pues su entorno familiar empieza a dar el espacio que precisa para que potencie su dimensión social. Por supuesto estas alteraciones generan en el niño una serie de estados que lo harán adaptarse poco a poco. De esta manera, la música juega un papel esencial en la vida social del sujeto. Pues por medio de estrategias tomadas en primera mano por el agente educativo, el proceso de socialización evidenciará los lazos que se empiezan a trazar generando acercamiento entre los estudiantes y su maestro, por medio del aprendizaje significativo que se enmarca en cada actividad.

El proceso de ingreso a la escolaridad resulta difícil para los niños, pues se enfrentan a la separación del hogar y el ingreso a un mundo completamente nuevo y desconocido, la labor del docente es actuar como facilitador en este proceso de adaptación. A partir del Análisis temático aplicado al trabajo de los estudiantes se determina como temáticas esenciales: Adaptación al contexto educativo, estrategias didácticas para la adaptación al entorno educativo, importancia del docente como mediador en situaciones de conflicto, creatividad pedagógica, innovación, impacto social, entre otros.

\section{Etapa de Reflexión}

Con el avance de dicho análisis se desarrolla una reescritura de lo expuesto y los significados implícitos en el texto de los estudiantes: El dato, da cuenta de un proceso amplio de conceptualización desarrollado por los estudiantes, proceso en el que además de realizar una exposición clara sobre la necesidad de comprender las características y necesidades de los niños y niñas al momento de enfrentarse a nuevos ambientes como lo son los educativos, presentan una propuesta innovadora para facilitar la adaptación de los niños a espacios que para ellos son desconocidos (Subcategoría: pertinencia pedagógica/innovación). Las estudiantes exponen ser capaces de llevar a la práctica la teoría aprendida en su formación académica, pues reconocen su papel como docentes, es su deben velar por el desarrollo adecuado y el aprendizaje de los niños (Subcategoría: Nuevos significados)

La Feria lúdica es diseñada como un espacio de disfrute, en el que se aprende y empodera en torno a la labor docente. Se encuentra explicito el reconocimiento de conceptos como infancia, lúdica, pedagogía, educación, didáctica, aprendizaje significativo, socialización, derechos de los niños (Subcategoría: Competencias a través de la formación académica). Con la estrategia lúdica planteada las estudiantes pretenden aportar a la deconstrucción de la enseñanza tradicional, implementando el juego guiado y estructurado para el aprendizaje pleno (Subcategoría: Experiencia pedagógica)

Mediante la puesta en marcha de la propuesta didáctica planteada las estudiantes dan aportes a la innovación y promoción de ambientes de aprendizaje para la interacción y relación social-personal con el medio, que son relaciones vitales para el crecimiento dentro de las habilidades y destrezas de la primera infancia (Subcategoría: Deconstrucción Infancias). De acuerdo con lo anterior, en la etapa de Reflexión se organizó la información se compendió la información en tablas, mediante las cuales se sistematizó la información y se reflexionó en torno a los hallazgos, a la luz de los contenidos curriculares del Seminario de Infancia y los alcances de la feria lúdico pedagógica, a partir de las unidades y las sub categorías de análisis. Hallazgos que se reunieron en un texto a modo de síntesis el cual se presenta a continuación:

\section{Hallazgos}

La reflexión en torno a las experiencias pedagógicas cotidianas, dentro y fuera del aula, demuestra que en los procesos de enseñanza aprendizaje, cada fenómeno pedagógico y didáctico, se encuentran un sinnúmero de significados: sociales, emocionales, éticos, económicos, objetivos y subjetivos, elementos con los cuales el docente debe contar y que le exigen actuar, crear, asumir y transformar de forma permanente, respondiendo a las circunstancias y las necesidades de los niños. Estos procesos se enmarcan y son posibles como un intercambio de saberes y experiencias, en los que el lenguaje y el reconocimiento de la diversidad y lo subjetivo es fundamental. 
Mediante el análisis de los trabajos, fotografías y videos resultado de la Feria Lúdico Pedagógica, se evidenció que los estudiantes implementan variedad de elementos para el desarrollo de sus actividades como son los disfraces, una escenografía adecuada, se toman el tiempo de diseñar actividades que buscan el aprendizaje activo y participativo, elemento de gran importancia pues la creatividad en el planeamiento y diseño de actividades es una destreza fundamental en el trabajo con infancia. Sin duda alguna el trabajo con infancia es una labor de total compromiso. Es fundamental que el educador de primera infancia esté con los niños y niñas, los acompañe y escuche. La cercanía y acompañamiento a los niños es un proceso que va en doble vía: el educador en formación enseña y a la vez aprende, así las educadoras en formación se hacen cercanas a los niños y niñas, acompañándolos en sus procesos, brindándoles seguridad y confianza. Se evidencia que asumen con compromiso el trabajo con infancia, reconociendo la importancia de su labor, yendo más allá integrándose a los espacios de aprendizaje y dinamizando la enseñanza. En el trabajo e interacción con infancias, la creatividad y la empatía son fundamentales, por lo que el conocimiento específico de conceptos como didáctica, lúdica, pedagogía y además de las políticas públicas de primera infancia tienen que hacer parte de los conocimientos del educador, en el trabajo con primera infancia el docente es un facilitador, debe promover espacios de interacción, debe conocer las políticas a favor de los niños reconociendo y construyendo contextos que propicien su buen desarrollo y respeto a sus derechos.

Por otra parte, el significado intrínseco del juego, es un elemento muy importante para deconstruir su concepto erróneo como actividad de simple esparcimiento, la actividad lúdica, la imaginación y la innovación deben ser asumidos como ejes que dinamizan el aprendizaje, así, el educador es sin duda la persona que más motivación debe tener al momento de compartir con los niños y niñas, dado el estado de desarrollo y la edad de los niños se hacen necesarias variedad de estrategias que promuevan la participación y acerquen a los educadores y los niños, por lo que el juego guiado es un enfoque de instrucción directa y a la vez una actividad libre, pues se organiza de acuerdo a un objetivo de aprendizaje, integra el medio ambiente al tiempo que permite a los niños mantener un alto grado de control sobre su aprendizaje.

De acuerdo con lo anterior, la persona que decide dedicarse al trabajo con infancias debe estar más que consiente de la importancia de su papel como acompañante, guía e intercesor entre el conocimiento a la curiosidad de los niños y niñas, pues los procesos educativos que diseña y dirige, deben propiciar el conocimiento, invitar a la reflexión y promover el pensamiento crítico, generando en los niños y niñas la consciencia de solucionar las problemáticas y transformar la sociedad, haciendo del quehacer educativo y la función docente en una "práctica creativa"

En los contenidos textuales se evidencia que las educadoras en formación se valen de sus experiencias de aprendizaje previas para educar, por lo que es fundamental que desde el programa se les dé un acompañamiento constante desde la academia, pues ellas son parte importante en la construcción de las generaciones futuras. Como elemento analítico de gran importancia, resalta el significado subyacente en cada uno de los trabajo y evidencia vivencial, que el educador de primera infancia es sin duda un actor social de gran importancia, mediante sus prácticas aporta a la transformación social, educa en valores y construye conocimiento integral, correspondiendo a los objetivos de la licenciatura en Pedagogía Infantil y en general de la Educación. Es tarea de la academia, dotar a los futuros educadores de primera infancia de las herramientas necesarias para derrumbar la estructura clásica del maestro como centro de conocimiento y el estudiante como sujeto pasivo y a la espera.

\section{DISCUSIÓN}

La investigación se realizó con el objetivo de reconocer la Feria lúdico Pedagógica como una estrategia de fortalecimiento, articulación y pertinencia curricular del programa de Licenciatura en Pedagogía Infantil de la Corporación UNIMINUTO y como un espacio que promueva la transformación y aporte a la dinamización de los actos educativos. De acuerdo con lo anterior, y retomando lo analizado en la investigación, resalta en los discursos y en las prácticas la necesidad de generar espacios en los que el educador más que ser quien imparte conocimientos, actúe como mediador entre los contenidos académicos y la curiosidad y necesidades de los niños y niñas. La práctica pedagógica ha de ser el espacio para que el pedagogo infantil en formación demuestre sus habilidades, destrezas y su creatividad para hacer del acto educativo un proceso dinámico, productivo, cercano que concuerde con los fines esenciales de la educación.

Mediante la metodología hermenéutica y elementos de análisis del enfoque fenomenológico, se profundizó en la subjetividad de los discursos y de las prácticas pedagógicas que de acuerdo con Van Manen (2003) citado por Galvis (2018) corresponde al "mundo de la vida, el mundo tal como lo experimentamos inmediatamente de un modo prereflexivo, y no tal como lo conceptualizamos o categorizamos, sino como reflexionamos sobre él". En este sentido, al ahondar en aspectos como la experiencia vivencial del quehacer educativo, se aporta a la reflexión en torno a lo que es la pedagogía, no como un elemento estable, sino como dinámico, que responde a las necesidades de los actores educativos y a los contextos donde se desarrolla, el acto educativo como un ambiente en el que confluyen múltiples subjetividades y formas de ver el mundo, 
pero no por ello carece de sentido y organización, sino que mantiene un orden en cuanto se mantiene en constante transformación, en un tipo de esencia inacabable y única.

La academia debe propender por brindar al licenciado en formación las herramientas teóricas y prácticas necesarias para que este tenga una visión clara sobre las necesidades de aprendizaje de los niños y niñas. De acuerdo con Galvis (2018), "El propósito no es generalizar conocimientos, probar hipótesis, elaborar leyes, establecer relaciones entre variables, etc. consiste en la profundización de los significados esenciales de la experiencia pedagógica. A nivel formal, el investigador llega a elaborar "una descripción (textual) estimulante y evocativa de las acciones, conductas, intenciones y experiencias humanas tal como las conocemos en el mundo de la vida" (Galvis 2018).

De acuerdo con los resultados de la investigación, se puede afirmar que la Feria Lúdico pedagógica es un espacio en el que se evidencian de forma práctica los alcances conceptuales de los contenidos curriculares del programa, en su ejecución los estudiantes llevan a la práctica lo aprendido en el transcurso del seminario en infancia, y a la vez integran y demuestran sus habilidades propias y conocimientos previos, exploran sus destrezas, su creatividad y definen su vocación, por otra parte, descubren herramientas útiles que aportan al desarrollo de su futura profesión y es que de acuerdo con Johnson et al. (2016): "Es deseable que los educadores de la primera infancia y los padres se alejen de las simples creencias sobre el juego hacia una conciencia más integral y una apreciación más reflexiva del tema. Con ese fin, hemos intentado integrar y compartir con el lector las opiniones contemporáneas de los principales académicos e investigadores interesados en el juego, el desarrollo de la primera infancia y la educación" (Christie y Yawkey 2016). La Feria Lúdico Pedagógica permite aprehender y desaprender, cuestiona, deconstruye y transforma concepciones y prácticas, devela sentidos intrínsecos en las acciones y discursos de los sujetos, para el caso los docentes en formación.

El presente estudio posibilitó develar sentidos y significados ocultos que aportan herramientas para mejorar las prácticas, las relaciones y los métodos de enseñanza- aprendizaje tanto en la formación de educadores como en el trabajo con infancia. De esta manera, sobresale la importancia de la experiencia lingüística, expresada en el texto, el cual genera la acción, y define lo que el ser humanos es. El texto es el resultado de la experiencia que narra sentido del mundo, "la comprensión que alcanza el hombre sobre sí mismo no puede realizarse si no es a través del lenguaje, puesto que el lenguaje configura la experiencia que tiene el hombre del mundo" (Ríos, 2013).

Por lo anterior, es importante que las experiencias y vivencias experimentadas por los docentes y estudiantes que participan la Feria lúdico pedagógica sean expresadas en textos, entrevistas y relatos para facilitar el análisis reflexivo de aspectos como la pedagogía, la didáctica el currículo, la enseñanza - aprendizaje, para definir las fortalezas y debilidades en el desarrollo de la misma, pues como afirma Ríos (2013): "el que narra su historia al mismo tiempo de comprender a sí mismo" (p. 55) a lo que se conoce como circulo hermenéutico de la comprensión. Existe una preocupación por desarrollar actividades que respondan a las necesidades de aprendizaje y a los contextos propios de los niños, así los estudiantes, dentro de sus discursos abordan temáticas como la inclusión, los Derechos Humanos y de los niños, el juego como herramienta pedagógica fundamental, la adaptación social mediante las artes, atención a la primera infancia, las cuales se enmarcan en las Políticas Públicas de Primera Infancia.

Sin duda alguna hay mucho por hacer, la Feria Lúdico Pedagógica, más que ser una muestra didáctica y un requisito del programa debe asumirse como un espacio a partir del cual reflexionar en torno al quehacer educativo en un mundo con tantas problemáticas y en el que la niñez es víctima de maltrato y abuso, es fundamental que la práctica pedagógica se asuma con seriedad, que el pedagogo en formación asuma con madurez su labor y comprenda que no es un cuidador sino un educador, un tutor y protector de los derechos de los niños, quien media entre el alcance de las metas y la llegada a ellas y el cumplimiento de objetivos; el docente en primera infancia además tiene en sus manos el futuro de las generaciones.

La reflexión sobre el quehacer educativo en la actualidad debe asumir el rol de derrumbar los paradigmas obsoletos y hacer del aprendizaje algo cercano y posible, dinámico y enriqueced. El docente en formación debe comprender que su labor educativa requiere de reflexión constante, pues a partir de la autoevaluación se pueden corregir posibles errores y se aporta a la dinamización de las prácticas pedagógicas.

Desde la academia debe comprender y asumir que la labor de formar educadores es una acción que aporta a la transformación social, por lo tanto, su deber es el de contextualizar las prácticas, reconocer la diversidad de los espacios y proceso educativos y dotar de herramientas claras y reflexivas a sus estudiantes par que sus enseñanzas trasciendan las aulas y promuevan la justicia social. El proceso investigativo desarrollado pretendió aportar un marco de reflexión de lo que desde las universidades se está aportando para la transformación social y la construcción y garantía de ambientes justos y respetuosos de los niños, por lo tanto, la reflexión sobre lo que es el quehacer pedagógico es una tarea inacabable y dinámica. 
Durante el trabajo realizado, evidenció que los proyectos desarrollados desde la UNIMINUTO, son una buena iniciativa de exposición de experiencias significativas en la formación de los menores, con bases didácticas, con el uso de la lúdica como herramienta de aprendizaje; esto ayuda a que se puedan comparar situaciones, diferentes o similares, según sea el caso, para entender a cabalidad, cual es el rol del docente en el proceso formativo del individuo, sobre todo en esta edad, y como, la universidad es la base de la construcción de personalidades de educadores acorde a las necesidades de la sociedad actualmente.

Sin embargo, el trabajo no puede quedar allí, debe ser socializado en conferencias y con el apoyo logístico propio de la comunidad educativa nacional e internacional, en la que se puedan ver los avances en materia pedagógica, sobre este tipo de temas, y poder ubicar a la UNIMINUTO en los primeros puestos de formación docente, en lo que concierne a la pedagogía infantil, y trasladar dichas situaciones a otras carreras dentro de la misma facultad, pues el problema que se genera de la negación de los otros actores dentro del debate de la educación, es que, el trabajo debe ser interdisciplinar y transversal

\section{CONCLUSIONES}

De acuerdo al trabajo presentado y a los resultados obtenidos, se pueden plantear las siguientes conclusiones principales:

1-. Sin duda alguna, la Feria lúdico Pedagógica constituye una estrategia funcional para fortalecer el programa de Licenciatura en Pedagogía Infantil de la Corporación UNIMINUTO y en general, las prácticas pedagógicas y procesos de enseñanza- aprendizaje de los docentes en formación.

2-. La lúdica como categoría fundamental, es una herramienta didáctica que dinamiza los procesos formativos en Primera Infancia.

3-. Son muchos los significados implícitos en el quehacer educativo con primera infancia, los cuales son el insumo principal para transformar y alimentar los planes, el currículo y los procesos formativos de futuros docentes de preescolar, más específicamente de la Corporación UNIMINUTO.

\section{REFERENCIAS}

Alvarado, N. J., Gestión curricular desde la visión del docente como constructor de currículo. REDINE, 11(1), 9-22. (2019). Broadhead, P., Howard, J. y Wood, E., Play and Learning in the Early Years: From Research to Practice. Edit. Arr. Lond. Eng. (2010).

Cáceres, M. F., Granada M. C., y Pomés, M. P., Inclusión y Juego en Infancia Temprana. Rev. Lat. Ed. Inc., 12(1), 181199. (2018).

Cremin, T. M., Creative teachers and creative teaching. Obt. Res. Gat.: ttps://www.researchgate.net/publication/48990754_Creative_teachers_and_creative_teaching (2015).

Galvis, S., La fenomenología hermenéutica en investigación: a propósito de un estudio sobre reflexión pedagógica desde las parábolas. Cu. Teo., X (1), 94-111. (2018)

Gardner, H. E., Estructuras de la mente. Teoría de las inteligencias múltiples. 3ra Ed. Fond. Cul. Eco. Méx. (2017).

Johnson, J.E., Christie, J. F., y Yawkey, T.D., Play and early childhood development. Sc.For. \& Co. Glen., IL, US. (2012)

Landreth, G.L., Play Therapy. The art of the relationship. 3rd Ed. Rout. N.Y. (2012)

López, P. I., El juego en la educación infantil y primaria. Autod. 19-37. (2017)

Ríos, T. J, La Hermenéutica reflexiva en la investigación educacional y social. Articulaciones entre Paul Ricoeur y la pedagogía crítica de Paulo Freire. Cent. Pubs. Un. Man. (2013)

Samacá, M. I., El espíritu científico en la Primera Infancia. Prax. \& Sab. 13(7), 89-106. (2015).

Sánchez, A.S., La importancia del juego en el proceso de enseñanza- aprendizaje de una lengua extranjera. Un. Vall. Pal, Esp. (2016).

Santos, M. L, y otros 4 autores, Aplicación de actividades lúdicas artísticas para el desarrollo del lenguaje de los niños y niñas .1ra ed. Ed. Grup. Comp. Gua., Ec. (2019).

Tapia, C. P., y Muñoz, P. Línea de vida como recurso narrativo para la formación socioemocional en estudiantes de pedagogía. https://dx.doi.org/10.19137/praxiseducativa-2019-230206 Prax. Ed., 23(2), 1-117. (2019).

Tresserras, A., Aportaciones a la formación del profesorado a través del análisis de una experiencia de arte y juego dramático en el primer ciclo de educación infantil. Eu. Her., Es.: Un. Pa. Va. (2017)

Wood, E., y Attfield, J., Play, Learning and the Early Childhood Curriculum. 3erd Ed. Saf. Pub. Inc. Ca., US, (2013). 
